\title{
Glueball-meson mixing
}

\author{
Vicente Vento ${ }^{\text {a }}$ \\ Departamento de Física Teórica and Instituto de Física Corpuscular, Universidad de Valencia - Consejo Superior \\ de Investigaciones Científicas, 46100 Burjassot (València), Spain
}

Received: 19 June 2015 / Revised: 13 November 2015

Published online: 7 January 2016

(C) The Author(s) 2016. This article is published with open access at Springerlink.com

Communicated by R. Alkofer

Abstract. Calculations in unquenched QCD for the scalar glueball spectrum have confirmed previous results of Gluodynamics finding a glueball at $\sim 1750 \mathrm{MeV}$. I analyze the implications of this discovery from the point of view of glueball-meson mixing in light of the experimental scalar sprectrum.

\section{Introduction}

Glueballs have not been an easy subject to study due to the lack of phenomenological support and therefore much debate has been associated with their properties [1]. I center the discussion here on the consequences of the spectrum obtained by lattice QCD where the results seem to be converging. In quenched QCD the masses of the scalar glueballs appear large $\geq 1700 \mathrm{MeV}[2-5]$, a result which has been confirmed by unquenched calculations [6].

Several glueball-meson mixing scenarios have been discussed in the literature using either lattice calculations or phenomenology [2,3,7-12]. I implement a combination of lattice results and phenomenology to study the implications of recent lattice results in the possible mixing scenarios in the scalar sector.

\section{The scalar spectrum}

Lattice QCD provides us with a value for the mass of the $0^{++}$glueball states as shown in table 1.

The three calculations give a similar mass for their lowest state for which I take the mean $1743 \pm 42 \mathrm{MeV}$ in my analysis.

In table 2, I show the experimental scalar spectrum, namely that of the particles labelled $f_{0}$.

The three heaviest states have not been confirmed and some authors also question the existence of the $f_{0}(1370)$.

An observation at the light of the experimental spectrum is that the excited glueballs obtained in QCD calculations are very high in mass and therefore I do not expect them to mix with the mesons. Thus the lattice calculations and the observed scalar spectrum lead to a scenario of one glueball amidst several scalar mesons in the range between $1-2 \mathrm{GeV}$.

\footnotetext{
a e-mail: vicente.vento@uv.es
}

Table 1. Glueball masses with $J^{P C}$ assignments. The column Gl reports the results of the unquenched QCD calculation by Gregory et al. [6], the columns Mp and Ky show the data from Morningstar and Peardon [4] and Chen et al. [5], respectively.

\begin{tabular}{|c|c|c|c|}
\hline \multirow{2}{*}{$J^{P C}$} & \multicolumn{3}{|c|}{ Mass MeV } \\
\cline { 2 - 4 } & Unquenched & \multicolumn{2}{|c|}{ Quenched } \\
\cline { 2 - 4 } & $\mathrm{Gl}$ & $\mathrm{Mp}$ & $\mathrm{Ky}$ \\
\hline $0^{++}$ & $1795(60)$ & $1730(50)(80)$ & $1710(50)(80)$ \\
\hline $0^{++}$ & $3760(240)$ & $2670(180)(130)$ & \\
\hline
\end{tabular}

\section{Glueball-meson mixing}

The most naive way of implementing a mixing scenario is by assuming a Hamiltonian which is not diagonal in the unmixed states. Thus there are two ingredients to this Hamiltonian, the unmixed masses and the off-diagonal mixing parameters.

The calculations thus far have led to different realizations of mixing. Some obtain that the $f_{0}(1710)$ is mostly glueball with small admixtures of $q \bar{q}$ states [2,3,9-11]. Others claim that the $f_{0}(1500)$ is mostly glueball, with small admixtures of $q \bar{q}$ states $[7,8]$.

I analyze next which scenarios could arise in a full QCD calculation. The idea is to construct a Hamiltonian which is not diagonal in the unmixed lattice states and diagonalize it to obtain the physical states. The decisive input is the glueball mass as given by lattice QCD. I assume that the rest of the particles in the unmixed Fock space are scalar mesons. Since the scalar meson spectrum of lattice QCD is not well known I will be guided by phenomenology. 
Table 2. Scalar particles appearing in the PDG's particle listings [13].

\begin{tabular}{|c|c|c|c|c|}
\hline$J^{P C}$ & Name & Mass MeV & Width MeV & Comment \\
\hline $0^{++}$ & $f_{0}(500)$ & $400-550$ & $400-700$ & \\
\hline $0^{++}$ & $f_{0}(980)$ & $990 \pm 20$ & $40-100$ & \\
\hline $0^{++}$ & $f_{0}(1370)$ & $1200-1500$ & $200-500$ & \\
\hline $0^{++}$ & $f_{0}(1500)$ & $1505 \pm 6$ & $109 \pm 7$ & \\
\hline $0^{++}$ & $f_{0}(1710)$ & $1720 \pm 6$ & $135 \pm 8$ & \\
\hline $0^{++}$ & $f_{0}(2020)$ & $1992 \pm 16$ & $442 \pm 60$ & needs confirmation \\
\hline $0^{++}$ & $f_{0}(2100)$ & $2013 \pm 8$ & $209 \pm 19$ & needs confirmation \\
\hline $0^{++}$ & $f_{0}(2200)$ & $2189 \pm 13$ & $238 \pm 50$ & needs confirmation \\
\hline
\end{tabular}

For the off-diagonal matrix elements I use Hamiltonian perturbation theory with $1 / N_{c}$ arguments. With these assumptions the matrix elements are of the form $\sim\left|\left\langle\Psi_{n}\left|H_{\text {non-diagonal }}\right| \Psi_{m}\right\rangle\right|^{2} /\left(E_{n}-E_{m}\right)$, therefore they depend on the inverse of the decay coupling $f_{\text {meson }}^{2} \sim N_{c}$, $f_{\text {glueball }}^{2} \sim N_{c}^{2}$, and on the inverse of the energy difference of the two levels. For almost degenerate unmixed states, the mixing parameters might be larger than for states of quite different masses.

For the off-diagonal matrix elements I use Hamiltonian perturbation theory with $1 / N_{c}$ arguments. With these assumptions the matrix elements are of the form $\sim\left|\left\langle\Psi_{n}\left|H_{\text {non-diagonal }}\right| \Psi_{m}\right\rangle\right|^{2} /\left(E_{n}-E_{m}\right)$, therefore they depend on the inverse of the decay coupling $f_{\text {meson }}^{2} \sim N_{c}$, $f_{\text {glueball }}^{2} \sim N_{c}^{2}$, and on the inverse of the energy difference of the two levels. For almost degenerate unmixed states, the mixing parameters might be larger than for states of quite different masses. I have assumed in this $N_{c}$ counting that the meson and glueball states can be degenerate, while not so the meson states. Thus the Hamiltonian matrix might look for a three-state mixing as

$$
\left(\begin{array}{ccc}
m_{s 1} & a & b \\
a & m_{s 2} & c \\
b & c & m_{g}
\end{array}\right)
$$

where, $m_{s 1}$ and $m_{s 2}$ represent the unmixed scalar meson masses, and $m_{g}$ the unmixed glueball mass. I assume no specific quark subtructure for the unmixed meson states. This is another result that lattice QCD must provide. Nonperturbative dynamics is so complex, as sum rule calculations have shown, that any specific input for the unmixed meson quark substructure might lead to misconceptions.

In this matrix, the diagonal matrix elements I take motivated by phenomenology and their values incorporate all higher-order corrections, $\sim 1+O\left(N_{c}^{-2}\right)+\ldots$ for the glueballs, and $\sim 1+O\left(N_{c}^{-1}\right)+\ldots$ for the mesons. The mixing matrix elements are $\sim O\left(N_{c}^{-2}\right)$ for meson-glueball states since $\Delta E \sim 1 / N_{c}$, but they are also $\sim O\left(N_{c}^{-2}\right)$ for the meson-meson states since $\Delta E \sim O(1)$ for them. Thus

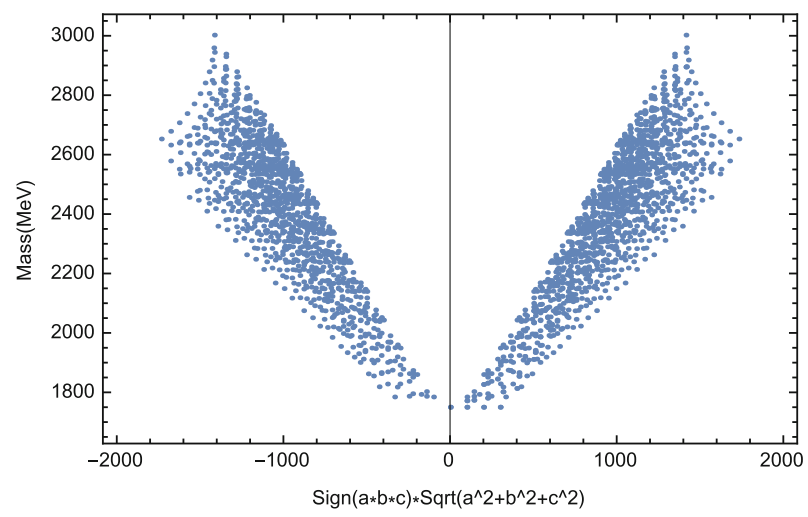

Fig. 1. The largest eigenvalue of the studied analog of a three state mixing matrix is shown as a function of the relevant parameters in Cardano's formulas. The values used for the diagonal elements were $1750,1500,1300$ close to the physical $f_{0}$ masses, and the mixing parameters $b, c$ range from $0 \rightarrow 1000$, and $a$ from $-1000 \rightarrow 1000$ in discrete steps.

$a, b$ and $c$ will be of the same order of magnitude, i.e. $\leq 250 \mathrm{MeV}$.

Field theoretic models give rise to mixing formulas which are quadratic in the hadronic masses [14,15]. The Hamiltonian formalism used here is linear in the masses and turns out more manageable for numerical analysis. For the values of the masses under study the difference between the two approaches is small.

It is trivial to prove that in a two by two symmetric mass mixing matrix the unmixed mass of the heavy state will increase after non-trivial diagonalisation. For a threestate mass mixing matrix it is not so trivial to show that the heaviest state mass also increases after non-trivial diagonalisation. This can be done using Cardano's formulas, as presented in fig. 1. Cardano's formulas depend only of one sign, $\operatorname{sign}(a b c)$, therefore one reaches all the desired values by only letting one of the parameters become negative. I have used as diagonal elements numbers close to the actual $f_{0}$ masses and the off diagonal elements have taken values far beyond my expectation for the physical mixing parameter values. Moreover, a full Cardano-Vieta analy- 

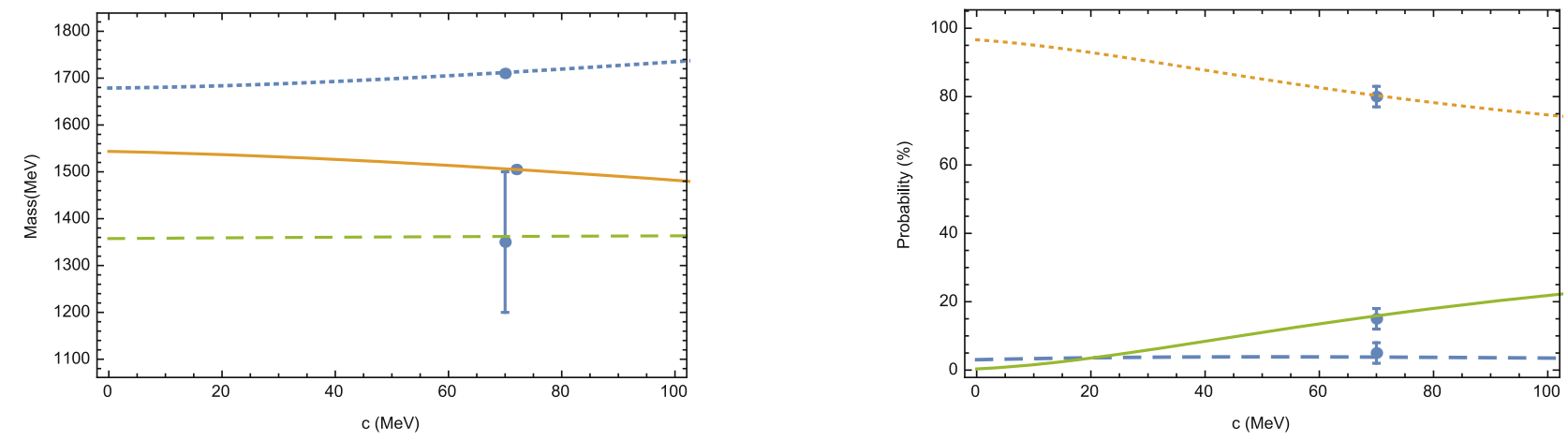

Fig. 2. Left: Eigenvalues of the mixing matrix for the glueball and two meson states. The masses and their errors are from PDG [13]. Right: The probability distribution for the $f_{0}(1710)$ of the unmixed states as a function of $c$, having fixed $a=b=$ $50 \mathrm{MeV}$. The dotted line represents the glueball with unmixed mass $(1670 \mathrm{MeV})$, the full line the heavy meson with unmixed mass $(1530 \mathrm{MeV})$, the dashed line the light meson with unmixed mass $(\sim 1380 \mathrm{MeV})$. The error bars in the probability curves are just to guide the eye.
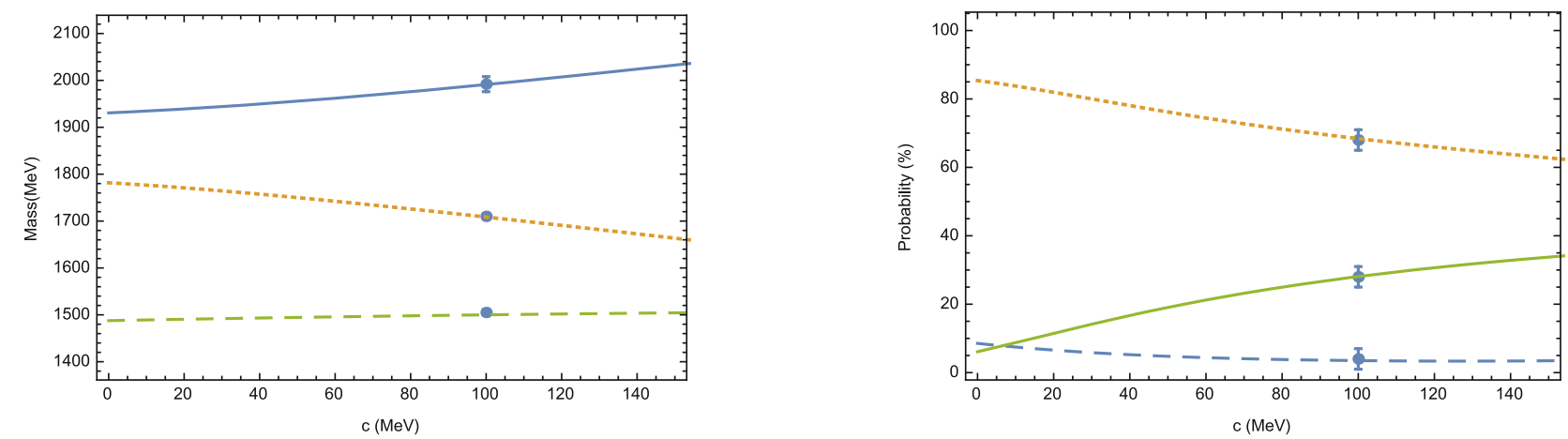

Fig. 3. Left: Eigenvalues of the mixing matrix for the glueball and two meson states. The masses and their errors are from PDG [13]. Right: The probability distribution for the $f_{0}(1710)$ of the unmixed states as a function of $c$. The other mixing parameters have been fixed to $a=100 \mathrm{MeV}$ and $b=100$. The dotted line represents the unmixed glueball (1750), the dashed line represents the light unmixed meson (1550), the full line represents the heavy unmixed meson (1900).

sis of the characteristic polynomial imposing the glueball mass and the physical meson masses leads to a much restricted range for the parameters $a, b$ and $c$, namely none of them can be larger than $250 \mathrm{MeV}$ in good agreement with our $1 / N_{c}$ analysis.

The QCD lattice mass value for the scalar glueball is very high and the error relatively small if the three values shown in table 1 are averaged with errors in quadrature $m_{g}=1743 \pm 42 \mathrm{MeV}$. One can still argue, given the errors in the lattice calculation, that the glueball mass could be below, but certainly not much below, the experimental $f_{0}(1710)$. Therefore, I foresee two scenarios: i) the unmixed QCD mass value is below the $f_{0}(1710)$ but close to it, and ii) the unmixed glueball mass value is above the $f_{0}(1710)$ in agreement with the lattice QCD average value. In the latter case I will analyze two cases depending on the unmixed spectrum, one will be associated with weak mixing, while the other with strong mixing.

The first scenario requires very small mixing, as can be seen in fig. 2 (left). The unmixed values were chosen $m_{s 1}=1380 \mathrm{MeV}, m_{s 2}=1530 \mathrm{MeV}$ and $m_{g}=1670 \mathrm{MeV}$, the latter two standard deviations below the central lattice value. The mixing parameters that fit the data are small $a=b=50 \mathrm{MeV}$ and $c=70 \mathrm{MeV}$. Note that in this case one can also trivially construct a two state weak mixing scenario, with the outcome that the $f_{0}(1710)$ is

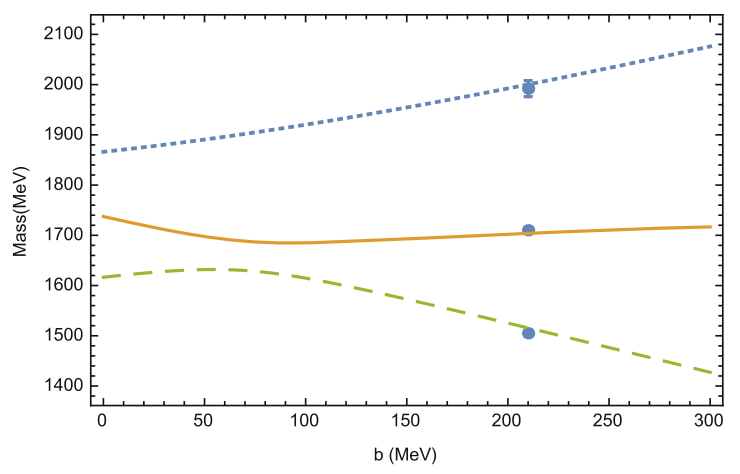

Fig. 4. Eigenvalues of the mixing matrix for the glueball and two meson states. The masses and their errors are from PDG [13].

again mostly glueball, the $f_{0}(1500)$ mostly meson and the mixing parameters are small. Thus the $f_{0}(1370)$ is not required from the point of view of mixing.

I show in fig. 2 (right) the probability distribution for the final mostly glueball state. It turns out that the experimental $f_{0}(1710)$ comes out mostly glueball in agreement with a lattice calculation of mixing [2,3], a lattice study of glueball decay [12], and other phenomenological analysis [11]. 

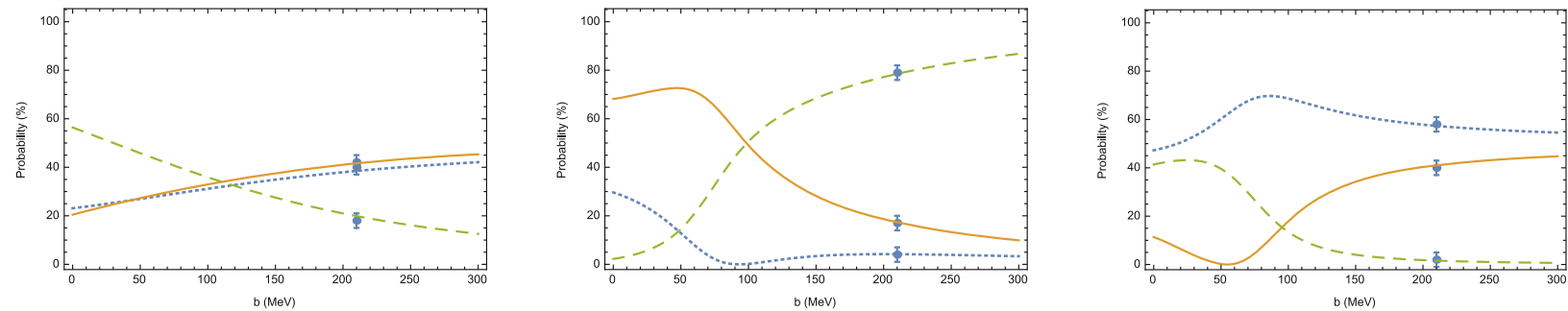

Fig. 5. The probability distributions as a function of $b$ of the mixed states, for mixing parameters $a=100 \mathrm{MeV}, c=70 \mathrm{MeV}$. The notation follows the unmixed states: the full line represents the glueball $(1750 \mathrm{MeV})$, the dashed line represents the meson $(1710 \mathrm{MeV})$, the dotted line represents the meson $(1760) \mathrm{MeV}$. The left figure represents the probability distribution of the $f_{0}(2020)$, the middle that of the $f_{0}(1710)$, and the right figure that of the $f_{0}(1500)$.

I must point out that none of the mixing parameters was fixed a priori. I performed the fit varying all the parameters within the allowed range. Once a good result was found I chose to plot against that parameter which was more sensitive to the mixing. The procedure will become particularly clear when the last scenario is shown.

Let me now elaborate on the other scenario, namely assuming $m_{g}>1710 \mathrm{MeV}$, i.e. I take $1750 \mathrm{MeV}$, close to the average value, as the unmixed glueball mass. As a result of Cardano's analysis, I know that the only way to get that mass down to the $f_{0}(1710)$ is by incorporating a heavier meson. In particular, I show in fig. 3 (left) a three state mixing where the $f_{0}(1710)$ is mostly glueball but the $f_{0}(2020)$, a not yet confirmed particle, must exist to get the experimental values for the lower masses. The corresponding probability curves, fig. 3 (right), show that the glueball component is at the level of $70 \%$. Most of the other $30 \%$ is carried by the $f_{0}(2020)$. Besides the existence of the $f_{0}(2020)$, another feature of this scheme is that the $f_{0}(1370)$, whatever it be, is not required, and thus the error bars are really very small and the fit quite restrictive. Another characteristic of this weak mixing scheme in the spread distribution of the unmixed spectrum: the particles appear with an energy step of $150-200 \mathrm{MeV}$. Note that in this case a two state mixing scenario could also be trivially constructed, but here the $f_{0}(2020)$ would be unavoidable and the $f_{0}(1500)$ would decouple from the mixing scheme.

Within the latter scenario also a strong mixing relization arises. As shown in fig. 4, I am able to get a good fit to the data starting from almost degenerate unmixed states. In this case the unmixed values are for two mesons at $1710 \mathrm{MeV}$ and $1760 \mathrm{MeV}$ and a glueball at $1750 \mathrm{MeV}$. The mixing parameters between the almost degenerate light meson and glueball is large $b=210 \mathrm{MeV}$, while the others are normal $a=100 \mathrm{MeV}, c=70 \mathrm{MeV}$. On the other hand this strong mixing affects the $f_{0}(1710)$ strongly, which now contains very little glueball, $\sim 17 \%$, while increasing dramatically the glueball content of the $f_{0}(1500), \sim 41 \%$, and some that of the $f_{0}(2020), \sim 42 \%$, see fig. 5. In this strong mixing scenario the glueball is a relic of the original glueball state and therefore it will be difficult to single out glueball properties. Again no need for the $f_{0}(1370)$ in the fit.

I might summarize at this stage my findings by stating that accepting a mass value for a glueball as obtained from lattice QCD for the unmixed glueball state and allowing for mixing under the strict scrutiny of the $1 / N_{c}$ expansion, two scenarios appear as dictated by the shape of the unmixed spectrum: i) a week mixing scenario, whose consequence is that the $f_{0}(1710)$ is an almost pure glueball state; ii) a strong mixing scenario in which the $f_{0}(1710)$ has almost no glueball component, while the $f_{0}(1500)$ and the $f_{0}(2020)$ have about $40 \%$ glueball component. The role played by the scalar meson spectrum in the realization of mixing is fundamental. A detailed lattice QCD description of this spectrum would determine the mixing scenario. Within the weak mixing scenario it is very important the value of the mass of the unmixed glueball state. If it is below the $f_{0}(1710)$ the mixing proceeds via the $f_{0}(1500)$ and the $f_{0}(1370)$ and it is not very constrained due to the large indeterminacy in the latter. If that mass is above $1710 \mathrm{MeV}$ the $f_{0}(2020)$ is required to bring the mass value down, while the $f_{0}(1370)$ is unnecessary.

In order to complete the analysis I should discuss decays. However, I cannot carry out that analysis because there is no dynamical model, as in refs. [14,15], behind the approach developed here. If lattice QCD would provide me with the decay rates of the glueball and the unmixed scalar mesons, I could a posteriori confirm which of the mixings scenarios leads to the experimental decay rates by using the mixing parameters obtained.

\section{Conclusions}

In this paper I take a very pragmatic point of view. I assume that the lowest found lattice QCD glueball state is associated with real physical states within the $f_{0}$ spectrum and try to establish the connection by implementing phenomenologically mixing with the nearby scalar mesons. In order to estimate the mixings I use the splitting pattern of the $f_{0}$ spectrum and $1 / N_{c}$ arguments.

I find two possible weak mixing scenarios which associate the lattice glueball mostly with the $f_{0}(1710)$. In this case the exact value of the unmixed glueball state is fundamental to determine the physical realization of mixing, being the $f_{0}(1710)$ the dominant scale. If the unmixed glueball mass is below that value, the $f_{0}(1370)$ should exist and may play a relevant role. If the mass is above, at least the $f_{0}(2020)$ is required to enter the mixing to bring down the unmixed mass to the physical mass. The scalar spectrum in both cases is loose, with an energy step between $150-200 \mathrm{MeV}$. 
I also find a strong mixing scenario which leads to the $f_{0}(1500)$ and the $f_{0}(2020)$ having a strong glueball component, while the $f_{0}(1710)$ is mostly mesonic in character. In this case the unmixed scalar spectrum presents an almost degeneracy around the unmixed glueball mass.

The main conclusion of this calculation is that given the fact that mixings are difficult to calculate in lattice QCD, a good knowledge of the scalar meson spectrum together with phenomenology will clarify the glueball constituency of the physical $f_{0}$ 's in full QCD. Once the scalar spectrum is known the $f_{0}$ spectrum and the arguments about the mixing used in this communication will fix quite strongly the mixing parameters.

I have presented a guide of possible mixing schemes as characterized by the structure of the spectrum, in particular the mass of the calculated scalar glueball has played a crucial role. Giving the above analysis I find that the $f_{0}(1710)$ being mostly glueball is the most natural scenario. This conclusion has been recently also reached by several authors using effective theories [16] and a holographic top-down approach $[17,18]$. If the glueball is accompanied, besides the $f_{0}(1500)$, by the $f_{0}(1370)$ or the $f_{0}(2020)$ in the mixing scenario is a matter of experimental determination. It is clear that for this purpose not only masses, but also decay rates should be used to determine the main properties of the glueball component. That study is outside the scope of this presentation due to lacking lattice QCD data.

Correspondence with Jaume Carbonell and Vincent Mathieu is gratefully acknowledged. This work was supported by the MINECO under contract FPA2013-47443-C2-1-P, by GVA-PROMETEOII/2014/066, SEV-2014-0398 and by CPAN(CSD-00042). I would like to thank the members of the TH-Unit at CERN for their hospitality.

Open Access This is an open access article distributed under the terms of the Creative Commons Attribution License (http://creativecommons.org/licenses/by/4.0), which permits unrestricted use, distribution, and reproduction in any medium, provided the original work is properly cited.

\section{References}

1. V. Mathieu, N. Kochelev, V. Vento, Int. J. Mod. Phys. E 18, 1 (2009) arXiv:0810.4453 [hep-ph].

2. W.J. Lee, D. Weingarten, Phys. Rev. D 61, 014015 (2000) hep-lat/9910008.

3. A. Vaccarino, D. Weingarten, Phys. Rev. D 60, 114501 (1999) hep-lat/9910007.

4. C.J. Morningstar, M.J. Peardon, Phys. Rev. D 60, 034509 (1999) hep-lat/9901004.

5. Y. Chen, A. Alexandru, S.J. Dong, T. Draper, I. Horvath, F.X. Lee, K.F. Liu, N. Mathur et al., Phys. Rev. D 73, 014516 (2006) hep-lat/0510074.

6. E. Gregory, A. Irving, B. Lucini, C. McNeile, A. Rago, C. Richards, E. Rinaldi, JHEP 10, 170 (2012) arXiv:1208.1858 [hep-lat].

7. C. Amsler, F.E. Close, Phys. Rev. D 53, 295 (1996) hepph/9507326.

8. WA102 Collaboration (D. Barberis et al.), Phys. Lett. B 397, 339 (1997).

9. M.K. Volkov, V.L. Yudichev, Eur. Phys. J. A 10, 223 (2001) hep-ph/0103003.

10. M.K. Volkov, V.L. Yudichev, Eur. Phys. J. A 10, 109 (2001).

11. H.Y. Cheng, Int. J. Mod. Phys. A 24, 3392 (2009) arXiv:0901.0741 [hep-ph].

12. CLQCD Collaboration (L.C. Gui et al.), Phys. Rev. Lett. 110, 021601 (2013) arXiv:1206.0125 [hep-lat].

13. Particle Data Group Collaboration (K.A. Olive et al.), Chin. Phys. C 38, 090001 (2014).

14. F. Giacosa, T. Gutsche, A. Faessler, Phys. Rev. C 71, 025202 (2005) hep-ph/0408085.

15. V. Mathieu, V. Vento, Phys. Rev. D 81, 034004 (2010) arXiv:0910.0212 [hep-ph].

16. S. Janowski, F. Giacosa, D.H. Rischke, Phys. Rev. D 90, 114005 (2014) arXiv:1408.4921 [hep-ph].

17. F. Brünner, D. Parganlija, A. Rebhan, Phys. Rev. D 91, 106002 (2015) arXiv:1501.07906 [hep-ph].

18. F. Brünner, A. Rebhan, arXiv:1504.05815 [hep-ph]. 